\title{
Managing the innovation supply chain to maximize personalized medicine.
}

\author{
Scott A. Waldman \\ Thomas Jefferson University \\ Andre Terzic \\ Mayo Clinic
}

Follow this and additional works at: https://jdc.jefferson.edu/petfp

Part of the Pharmacy and Pharmaceutical Sciences Commons Let us know how access to this document benefits you

\section{Recommended Citation}

Waldman, Scott A. and Terzic, Andre, "Managing the innovation supply chain to maximize personalized medicine." (2014). Department of Pharmacology and Experimental Therapeutics Faculty Papers. Paper 59.

https://jdc.jefferson.edu/petfp/59

This Article is brought to you for free and open access by the Jefferson Digital Commons. The Jefferson Digital Commons is a service of Thomas Jefferson University's Center for Teaching and Learning (CTL). The Commons is a showcase for Jefferson books and journals, peer-reviewed scholarly publications, unique historical collections from the University archives, and teaching tools. The Jefferson Digital Commons allows researchers and interested readers anywhere in the world to learn about and keep up to date with Jefferson scholarship. This article has been accepted for inclusion in Department of Pharmacology and Experimental Therapeutics Faculty Papers by an authorized administrator of the Jefferson Digital Commons. For more information, please contact: JeffersonDigitalCommons@jefferson.edu. 


\title{
Managing the Innovation Supply Chain to Maximize Personalized Medicine
}

\author{
SA Waldman ${ }^{1}$ and A Terzic ${ }^{2}$
}

${ }^{1}$ Delaware Valley Institute for Clinical and Translational Science, Department of Pharmacology and Experimental Therapeutics, Division of Clinical Pharmacology, Department of Medicine, Thomas Jefferson University, Philadelphia, Pennsylvania, USA;

and

${ }^{2}$ Mayo Clinic Center for Regenerative Medicine, Divisions of Cardiovascular Diseases and Clinical Pharmacology, Departments of Medicine, Molecular Pharmacology and Experimental Therapeutics and Medical Genetics, Mayo Clinic, Rochester, Minnesota, USA

\section{Correspondence}

Scott A. Waldman, MD, PhD,

Thomas Jefferson University

132 South $10^{\text {th }}$ Street, 1170 Main

Philadelphia, PA 19107

scott.waldman@jefferson.edu

and

Andre Terzic, MD, PhD,

Mayo Clinic

200, First Street SW, Stabile 5

Rochester, MN 55905

terzic.andre@mayo.edu

Title:

Words in Abstract:

70 characters (with spaces)

Body text word count: 1,775

References:

182

Figures:

34

Tables: 
Personalized medicine epitomizes an evolving model of care tailored to the individual patient. This emerging paradigm harnesses radical technological advances to define molecular characteristics of each patient and decipher their unique pathophysiological processes. Translated into individualized algorithms, the practice of personalized medicine aims to predict, prevent and cure disease without afflicting therapeutic adverse events. While the transformative power of personalized medicine is generally recognized by physicians, patients and payers, the complexity of translating discoveries into new modalities that transform healthcare is less appreciated. We often consider the flow of innovation and technology along a continuum of discovery, development, regulation, and application bridging the bench with the bedside. However, this process also can be viewed through a complementary prism, as a necessary supply chain of services and providers, each making essential contributions to the development of the final product to maximize value to consumers. Considering personalized medicine in this context of supply chain management highlights essential points of vulnerability and/or scalability that can ultimately constrain or potentiate translation of the biological revolution into individualized diagnostics and therapeutics for optimized value creation and delivery. 


\section{The whole is greater than the sum of the parts - Aristotle}

It is generally appreciated that the revolution in biology is transforming healthcare from one-size-fits-all therapeutics, to individual tailored disease management. ${ }^{1}, 2$ Science and medicine are increasingly providing molecular solutions that offer unprecedented opportunities to intervene in disease risk, prevention, prognosis and cure. $^{3}$ Emerging technology platforms provide diagnostics that identify corruption of individual molecular circuits disrupting signaling networks and organismal ecosystems which can be repaired through genomic, molecular or regenerative interventions specifically tailored to individual pathophysiology. ${ }^{4}$ This scientific revolution drives the development of personalized medicine, which exploits insights in molecular pathology to generate tailored mechanism-based therapeutics and enhance their curative effectiveness, while minimizing adverse events. ${ }^{5}$ The expanding toolbox of gene-based, molecular, omic, and regenerative technologies offers unparalleled opportunities to personalize diagnostics and therapeutics that can be applied across diseases, ethnicities, and geographies. ., $^{6}$

The fruits of this revolution in creating personalized diagnostic and therapeutic products are generally recognized by patients and their healthcare providers. ${ }^{7,} 8$ What may be less appreciated is the complexity of processes leading from laboratory-based discovery to individualized patient management algorithms in practice. We typically consider the science of translational therapeutics in discreet sequential steps in which the flow of information and technology moves from left to right along a continuum, from fundamental discovery of molecular principles and therapeutic targets, to development involving clinical trials that prove efficacy and safety, through regulatory approval that certifies the utility of the management approach, to application in patients and populations - the "DDRU continuum". 3, 9-12 This model broadly categorizes the stages translating invention into practice. However, it obscures the integral contributions by individual practitioners and domain-specific experts, and the impact of the external 
environment, which shape the process at every phase, from discovery to application. Without considering these individual components, their contributions, and their relationship to the over-arching continuum, we risk under-estimating points of vulnerability which can constrain or potentiate the innovation that serves as the lifeblood of personalized medicine.

It is useful here to borrow a page from the manufacturing sciences to consider the advanced product development continuum in the context of supply chain management. In this model, the movement and storage of raw materials, work-inprocess inventories, and finished products from the point of origin to the point of use is strategically managed to create net value with a competitive infrastructure, synchronizing supply with demand, and measuring performance globally across the entire enterprise. ${ }^{13}$ Indeed, supply chain strategies require a total systems view of the links in the chain that work together efficiently to create customer satisfaction at the endpoint of product delivery to the consumer. Efficiencies must be maximized at each of the component steps to produce added value across the entire continuum to optimize the benefit to the end user. This concept focuses on total systems efficiency to produce optimum value by generating the best product while minimizing waste and inefficiency. In this model, the supply chain is the set of organizations and processes linked by one or more upstream and downstream flows of products or information from a source to an end user.

By analogy, the DDRU continuum can be considered a type of product development/supply chain, with the raw materials of intellectual innovation in combination with specimens, reagents, and biologicals undergoing transformation by discovery scientists, clinical trialists, regulatory scientists and clinicians into integrated diagnostic and therapeutic paradigms that embody personalized medicine for end users including patients, their healthcare providers, and payers. The essential nature of raw materials to this supply chain is exemplified by the Commentary by Lu and Flockhart, 
who propose a national program to collect annotated biospecimens, similar to the national model for organ donor identification. These authors underscore the critical vulnerability of the DDRU continuum and innovation in individualized medicine in the context of limitations in the availability of biospecimens accompanied by full clinical and demographic annotation. Indeed, in the absence of this essential national resource, the ability to translate novel genomic observations identified in the laboratory into useful diagnostic and therapeutic targets that can be applied to disease-specific tailoring of patient management will undergo progressively greater levels of constraint. These constraints reflect an inability to validate and confirm clinical utility of the many genomic associations through standard prospective multicenter blinded clinical trials, which are logistically and economically infeasible. Rather, retrospective validation of clinical utility employing annotated biospecimens from a national repository represents an adaptive solution that creatively aligns resources with needs to maximize value of discoveries to end users of personalized medicine while minimizing costs and inefficiencies.

Moving along the supply chain, laboratory-based investigations are using those essential biospecimens in conjunction with emerging technology platforms to create molecular diagnostic products that are intrinsic components of the personalized medicine paradigm. ${ }^{12,14}$ These diagnostics identify pathophysiological biomolecules used for disease prediction, risk assessment, prognosis, and diagnosis. Further, these diagnostics can identify genetic variations in patients creating susceptibility to adverse therapeutic events. In his Commentary, Kesselheim highlights the recent Supreme Court decision against the patentability of natural processes. ${ }^{15}$ For example, developing a test identifying genetic variants that render patients unable to metabolize a drug, putting them at risk for toxic adverse events, is ineligible for patentability. Similarly, tests that define genetic mutations providing prognostic information predicting disease outcome also cannot be protected by patents. Here, policy decisions external to the scientific process may negatively shape the supply chain of innovation available to 
expand personalized medicine. Indeed, the absence of patent protection for these, and related, discoveries creates an economic vulnerability in the innovation supply chain, reflecting the absence of a period of market exclusivity for these discoveries characteristically granted through patent protection. This economic vulnerability threatens the flow of innovation by constraining investment in the development of intellectual property that cannot be patent protected. This absence of market exclusivity could strangle the supply of novel diagnostic products that are essential for downstream processes translating scientific innovation into the clinical tools supporting the evolution of personalized medicine.

The negative impact of these policy decisions concerning intellectual property stands as a striking counterpoint to the innovation in pharmacometabolomics underscored by Kaddurah-Daouk and her colleagues. ${ }^{16}$ This State of the Art review highlights the transformative potential of the study of metabolism at the omic level, to reveal novel molecular mechanisms underlying inter-individual variability in pathophysiology, drug action, and the evolution of adverse responses to therapeutics. In turn, these emerging mechanistic insights will form the basis for novel diagnostic tests based on metabolic signatures of patients that can specifically target individualized therapeutic approaches, to expand the supply chain of innovation feeding the personalized medicine paradigm. ${ }^{17-21}$ Similarly, Momper and Wagner in their Development remind us of the essential utility of therapeutic drug monitoring as a cornerstone of classical clinical pharmacology practice which has its origins in the pre-genomic era. ${ }^{22}$ This approach continues to provide unique insights into inter-individual variability in therapeutic responses and adverse events to drugs, especially in cohorts that exhibit highly dynamic metabolic phenotypes, like the pediatric population. Indeed, co-development of companion drug monitoring algorithms that accompany novel therapeutic approaches has the potential to dampen the variability in therapeutic and adverse responses to interventions, generally, in patient populations, suggesting a position for these approaches in strategies of supply chain management associated with the development of emerging targeted therapies. ${ }^{22}$ In 
that context, it will be important to monitor whether the recent Supreme Court decisions on intellectual property ${ }^{15}$ have a negative impact on the advancement of pharmacometabolomics or companion therapeutic drug monitoring approaches as novel diagnostics driving innovation in personalized medicine.

Toward the other end of the supply chain, there are emerging challenges in confirming the utility of novel diagnostic and therapeutic approaches at the center of personalized medicine. Selker and his colleagues highlight in their State of the Art the potential for standard clinical development approaches to over-estimate the utility of emerging paradigms in personalized medicine in general clinical practice. As these emerging approaches embrace higher orders of molecular specificity and patient individualization, there is a requirement for standard phase III efficacy trial designs to use highly homogenous populations of patients carrying the molecular target of interest to reveal clinical efficacy. In that context, efficacy revealed in these trials may be sufficient to achieve regulatory approval, but may not translate into true effectiveness in actual clinical practice, where patients are highly heterogeneous with respect to genotypes and phenotypes, and environments and demographics cannot be closely defined or controlled. In order to improve the flow of novel technological advancements along the supply chain from the point of innovation in the laboratory to end users like patients, healthcare providers and payers, Selker and his colleagues suggest evolving the standard phase III paradigm into efficacy-effectiveness trials. ${ }^{22}$ In this model, therapeutic interventions successfully completing standard phase III efficacy trials in narrowly defined patient populations would immediately transition into effectiveness trials to estimate the true utility of these approaches in heterogeneous populations that constitute actual medical practice.

Finally, at the very end of the supply chain, the deluge of omic information, its relationship to clinical practice, and its utility in maximizing improvements in patient outcomes while minimizing healthcare costs continues to remain a conundrum to patients, providers and payers. What are the criteria that support the utility of specific omic platforms in patient management paradigms? How should patients and providers 
assess those criteria for their utility, and how should payers assess their ratio of cost to benefit? It is readily apparent that without solving this conundrum, the supply chain transforming invention and innovation into end user value will prematurely terminate. In their State of the Art review, Dotson and colleagues offer one $e^{23}$ of a number ${ }^{24-34}$ of algorithms to assess the evidence base supporting implementation of genomic information into clinical practice and patient management.

Personalized medicine is having a transformative impact on disease risk assessment, prediction, prevention, prognosis and cure. ${ }^{9,}{ }^{11}$ The present discussion highlights the operational flow of technology along a supply chain of innovation, from laboratory to end users, which requires management at each individual step to maximize healthcare and economic benefits. Our challenge is to establish the policies and processes that become an engine driving innovation through this cascade, rather than a weight dragging down the system. 


\section{ACKNOWLEDGEMENTS}

SAW is the Samuel M.V. Hamilton Endowed Professor of Thomas Jefferson University. AT is Michael S. and Mary Sue Shannon Family Director, Center for Regenerative Medicine, and Marriott Family Professor of Cardiovascular Research at Mayo Clinic. This work was supported by grants from NIH (CA146033; CA170533), the Pennsylvania Department of Health, Targeted Diagnostic \& Therapeutics, Inc, and Mayo Clinic. The Pennsylvania Department of Health specifically disclaims responsibility for any analyses, interpretations or conclusions.

\section{FINANCIAL DISCLOSURES}

The authors have no relevant disclosures. 


\section{REFERENCES}

(1) Waldman, S.A. \& Terzic, A. Systems-based discovery advances drug development. Clin Pharmacol Ther 93, 285-7 (2013).

(2) Evans, W.E., Crews, K.R. \& Pui, C.H. A health-care system perspective on implementing genomic medicine: pediatric acute lymphoblastic leukemia as a paradigm. Clin Pharmacol Ther 94, 224-9 (2013).

(3) Waldman, S.A. \& Terzic, A. Information hierarchies optimize patient-centered solutions. Clin Pharmacol Ther 93, 3-7 (2013).

(4) Gonzalez de Castro, D., Clarke, P.A., Al-Lazikani, B. \& Workman, P. Personalized cancer medicine: molecular diagnostics, predictive biomarkers, and drug resistance. Clin Pharmacol Ther 93, 252-9 (2013).

(5) Jacunski, A. \& Tatonetti, N.P. Connecting the Dots: Applications of Network Medicine in Pharmacology and Disease. Clin Pharmacol Ther, (2013).

(6) Lewis, J.P., Yerges-Armstrong, L.M., Ellero-Simatos, S., Georgiades, A., Kaddurah-Daouk, R. \& Hankemeier, T. Integration of Pharmacometabolomic and Pharmacogenomic Approaches Reveals Novel Insights Into Antiplatelet Therapy. Clin Pharmacol Ther, (2013).

(7) Roden, D.M. \& Tyndale, R.F. Genomic medicine, precision medicine, personalized medicine: what's in a name? Clin Pharmacol Ther 94, 169-72 (2013).

(8) Johnson, J.A., Klein, T.E. \& Relling, M.V. Clinical implementation of pharmacogenetics: more than one gene at a time. Clin Pharmacol Ther 93, 3845 (2013). 
(9) Waldman, S.A. \& Terzic, A. The value proposition of molecular medicine. Clin Trans/ Sci 5, 108-10 (2012).

(10) Waldman, S.A. \& Terzic, A. Knowledge cycle transforms therapeutic innovation. Clin Pharmacol Ther 91, 3-8 (2012).

(11) Waldman, S.A. \& Terzic, A. Translational medicine individualizes healthcare discovery, development and delivery. Foreword. Biomark Med 7, 1-3 (2013).

(12) Waldman, S.A., van der Graaf, P.H. \& Terzic, A. Systems approaches evolve clinical pharmacology. CPT Pharmacometrics Syst Pharmacol 2, e68 (2013).

(13) Harland, C.M. Supply chain management, purchasing and supply management, logistics, vertical integration, materials management, and supply chain dynamics. In: Blackwell Encyclopedia Dictionary of Operations Management (ed. Slack, N.) (Blackwell, United Kingdom, 1996).

(14) Whirl-Carrillo, M. et al. Pharmacogenomics knowledge for personalized medicine. Clin Pharmacol Ther 92, 414-7 (2012).

(15) Kesselheim, A.S., (2014).

(16) Kaddurah-Daouk, R., (2014).

(17) James, L.P. Metabolomics: integration of a new "omics" with clinical pharmacology. Clin Pharmacol Ther 94, 547-51 (2013).

(18) Kaddurah-Daouk, R. \& Weinshilboum, R.M. Pharmacometabolomics: Implications for Clinical Pharmacology and Systems Pharmacology. Clin Pharmacol Ther, (2013).

(19) Krauss, R.M., Zhu, H. \& Kaddurah-Daouk, R. Pharmacometabolomics of statin response. Clin Pharmacol Ther 94, 562-5 (2013). 
(20) Robertson, D.G. \& Frevert, U. Metabolomics in drug discovery and development. Clin Pharmacol Ther 94, 559-61 (2013).

(21) Shin, K.H., Choi, M.H., Lim, K.S., Yu, K.S., Jang, I.J. \& Cho, J.Y. Evaluation of Endogenous Metabolic Markers of Hepatic CYP3A Activity Using Metabolic Profiling and Midazolam Clearance. Clin Pharmacol Ther 94, 601-9 (2013).

(22) Wagner. (2014).

(23) Dotson. (2014).

(24) Caudle, K.E. et al. Clinical Pharmacogenetics Implementation Consortium Guidelines for Dihydropyrimidine Dehydrogenase Genotype and Fluoropyrimidine Dosing. Clin Pharmacol Ther, (2013).

(25) Hershfield, M.S. et al. Clinical Pharmacogenetics Implementation Consortium guidelines for human leukocyte antigen-B genotype and allopurinol dosing. Clin Pharmacol Ther 93, 153-8 (2012).

(26) Hicks, J.K. et al. Clinical Pharmacogenetics Implementation Consortium guideline for CYP2D6 and CYP2C19 genotypes and dosing of tricyclic antidepressants. Clin Pharmacol Ther 93, $402-8$ (2013).

(27) Leckband, S.G. et al. Clinical pharmacogenetics implementation consortium guidelines for HLA-B genotype and carbamazepine dosing. Clin Pharmacol Ther 94, 324-8 (2013).

(28) Martin, M.A., Klein, T.E., Dong, B.J., Pirmohamed, M., Haas, D.W. \& Kroetz, D.L. Clinical pharmacogenetics implementation consortium guidelines for HLA-B genotype and abacavir dosing. Clin Pharmacol Ther 91, 734-8 (2012). 
(29) Muir, A.J. et al. Clinical Pharmacogenetics Implementation Consortium (CPIC) guidelines for IFNL3 (IL28B) genotype and peginterferon alpha based regimens. Clin Pharmacol Ther, (2013).

(30) Relling, M.V. et al. Clinical pharmacogenetics implementation consortium guidelines for thiopurine methyltransferase genotype and thiopurine dosing: 2013 update. Clin Pharmacol Ther 93, 324-5 (2013).

(31) Relling, M.V. \& Klein, T.E. CPIC: Clinical Pharmacogenetics Implementation Consortium of the Pharmacogenomics Research Network. Clin Pharmacol Ther 89, 464-7 (2011).

(32) Scott, S.A. et al. Clinical pharmacogenetics implementation consortium guidelines for CYP2C19 genotype and clopidogrel therapy: 2013 update. Clin Pharmacol Ther 94, 317-23 (2013).

(33) Shuldiner, A.R. et al. The Pharmacogenomics Research Network Translational Pharmacogenetics Program: overcoming challenges of real-world implementation. Clin Pharmacol Ther 94, 207-10 (2013).

(34) Wilke, R.A. et al. The clinical pharmacogenomics implementation consortium: CPIC guideline for SLCO1B1 and simvastatin-induced myopathy. Clin Pharmacol Ther 92, 112-7 (2012). 